\title{
Origami fold states: concept and design tool
}

\author{
Alex Avila ${ }^{1}$, Spencer P. Magleby ${ }^{1}$, Robert J. Lang ${ }^{2}$, and Larry L. Howell ${ }^{1}$ \\ ${ }^{1}$ Brigham Young University,Provo, Utah 84602, USA \\ ${ }^{2}$ Lang Origami, Alamo, CA 94507, USA \\ Correspondence: Alex Avila (alexavila@byu.net)
}

Received: 27 September 2018 - Accepted: 12 February 2019 - Published: 4 March 2019

\begin{abstract}
The ability of origami to alter its properties and behaviors with its shape makes it an elegant source of inspiration for many engineering designs challenges. Fold states specify the shape of the origami - its facets, creases, and fold angles. Origami research recognizes several acknowledged fold states: the unfolded, fully folded, and flat-folded states. However, these fold states are not comprehensive, excluding some of the most predominant fold states in origami-based devices. In this paper we propose a comprehensive list of fold states based on fold angles. We support the method of categorizing fold states by evaluating the functions and fold states of a large sample of origami-based devices. These correlations provide insight for selecting fold states for origami-based design. We discuss properties and behaviors of the fold states individually and provide a process for fold-state selection.
\end{abstract}

\section{Introduction}

Origami has become an effective source of inspiration for engineering solutions. The scalable nature of origami, its inherent motion, and potential for reconfigurability make its influence versatile and applicable in many fields. Origami has inspired a range of innovations including an origamibased helmet for emergencies (Yang et al., 2017), an unfolding telescope for space exploration (Early et al., 2003), and re-configurable origami furniture for homes (Lin, 2011).

One reason origami is an elegant and natural source for solutions to many engineering challenges is that an origamibased device has different properties and behaviors in each fold state - an arrangement of the facets and creases along with its fold angles. The different properties of each fold state, such as shape, dimensions, and projected surface area, allow a device based on a single origami crease pattern to perform multiple functions. For example, an origami-based ballistic barrier was designed for storage and transportation in one fold state and then partially unfolded to provide ballistic protection in another fold state (see Fig. 1) (Seymour et al., 2018).

Specific origami fold states, such as the unfolded and flatfolded states, have garnered interest in the artistic and mathematic fields because of their unique properties and behaviors. The properties and behaviors of these fold states make them especially useful for certain engineering applications. For example, engineers use unfolded states for planar manufacturing and flat-folded states for storage or transportation of devices.

While researchers have studied the unfolded and flatfolded states extensively, and engineers have used them in various devices (Bern and Hayes, 1996; Rabinovich et al., 2018; Lang, 2017; Ku and Demaine, 2016), there are many other fold states that occur in origami art and origami-based devices. For example, the origami starshade is not completely flat-folded when it is stowed (Lo, 2009), and the origamibased ballistic barrier is not deployed completely flat. There are no widely accepted terms that refer to these fold states.

Lacking a comprehensive list of fold states, designers limit their ability to discuss many common fold states and risk neglecting fold states when designing origami-based devices.

In this paper, we form a list of seven types of origami fold states. Examples of origami-based devices for each type of fold state are identified and discussed, along with their properties and behaviors. We demonstrate how fold-state types can be used in origami-based design. The terms, examples, and tables presented in this paper offer a way to discuss origami fold states more thoroughly, identify uses for each fold state, and design origami-based devices. 


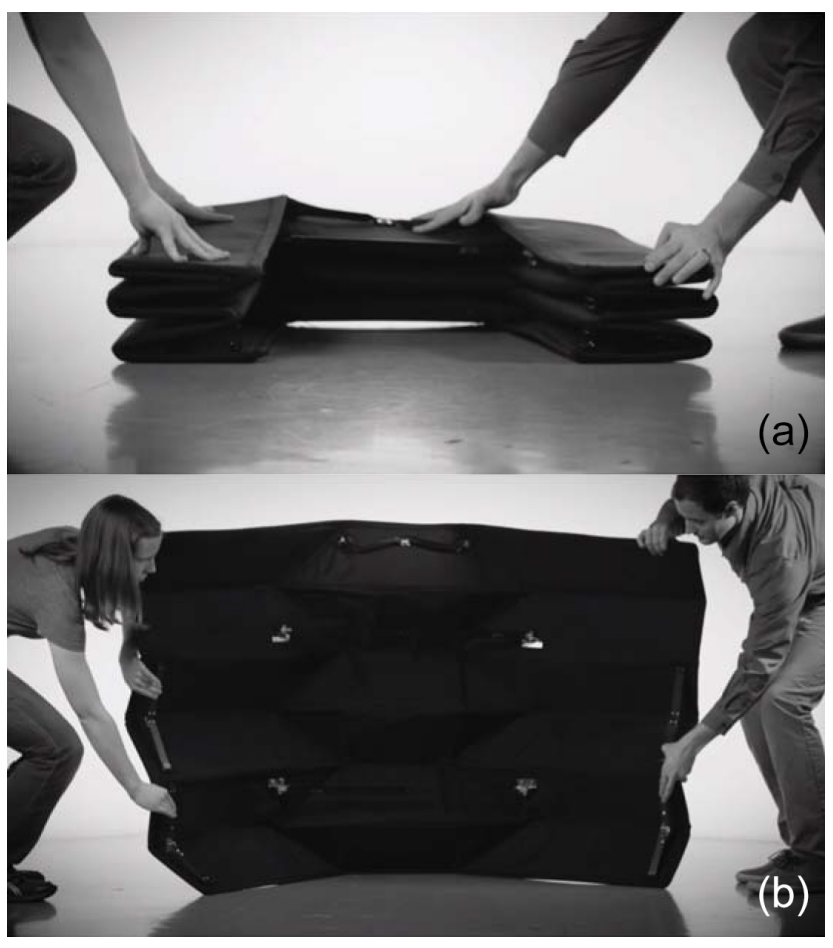

Figure 1. An origami-based ballistic shield in its closed state (a) and deployed state (b).

\section{Definitions}

Origami nomenclature is an eclectic assortment of artistic, mathematical, and engineering terms (Lang, 2017; Greenberg et al., 2011; Tachi, 2010). Most terms are clearly defined, however, some are used inconsistently. For example, origami in the fully folded state can refer to origami that (1) can no longer fold in a fold direction (Wu and You, 2010), (2) is folded flat (all the fold angles are -180 or $180^{\circ}$ ) (Pagano et al., 2017), or (3) is in its final fold state irrespective of its fold angles (Kuribayashi et al., 2006).

To create a comprehensive and consistent list of fold types, we must first define fold-angle sets and other ancillary terms. The first section contains those foundational definitions, and the second section defines the specific fold types and fold state types.

The parenthesis and braces used in the definitions and in the rest of the paper follow list and set notation. As well the facets are assumed to have zero thickness.

\subsection{Foundational definitions for fold types}

A crease is a linear feature along which a fold takes place. A crease can be unfolded, partially folded, or fully (flat) folded (Lang, 2017). While the term crease typically means a revolute hinge joint formed from material deformation, we use the term crease to refer to any revolute-like joint in an

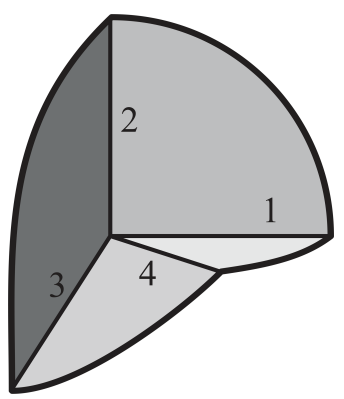

Figure 2. A degree-4 vertex shown in a partially folded state (Lang, $2017)$. It has a complete fold-angle set of $\left(\left(1,-90^{\circ}\right),\left(2,-135^{\circ}\right)\right.$, $\left.\left(3,-90^{\circ}\right),\left(4,135^{\circ}\right)\right)$.

origami-based device, e.g. hinge joints, compliant joints, and rolling contact joints.

A fold is a crease with an associated fold angle or assignment (mountain or valley).

A fold angle is the signed angle between the normal vectors of two facets that meet at a fold (Lang, 2017).

An origami fold state or a fold state is a 2-D or 3-D arrangement of facets that are connected to each other by folds and vertices, plus any layer order information for facets that are pairwise coplanar.

Terms, such as origami configuration, origami figure, and folded form, have similar meanings with origami fold state, but in this paper we will use the term fold state exclusively.

A fold-angle set is any subset of the fold angles of a fold state, together with the mapping between fold angle and fold. The fold number and corresponding fold angle are listed as a pairs. For example, in Fig. 2, one fold-angle set of the fold state is $\left(\left(1,-90^{\circ}\right),\left(3,-90^{\circ}\right),\left(4,135^{\circ}\right)\right)$.

A complete fold-angle set of a fold state is a fold-angle set that includes all folds of the fold state. The mapping of the fold angle with a fold can be implicit. For example, rather than writing the complete fold-angle set of the fold state in Fig. 2 as $\left(\left(1,-90^{\circ}\right),\left(2,135^{\circ}\right),\left(3,-90^{\circ}\right),\left(4,135^{\circ}\right)\right)$ we can write it as $\left(-90^{\circ}, 135^{\circ},-90^{\circ}, 135^{\circ}\right)$.

\subsection{Fold types}

A fold type is a property that conveys information about the value(s) of folds angles, fold-angle sets, or fold states. We specify whether a fold type refers to a fold, fold-angle set, or fold state by including it in the term, e.g. fold-angle type, fold-angle set type, fold-state type.

An angle or a fold-angle set is unfolded (U) if the angle or all angles in the set are in the set $U \equiv\left\{0^{\circ}\right\}$.

An angle or a fold-angle set is partially folded (P) if all angles in the set are in $P \equiv\left(-180^{\circ}, 180^{\circ}\right) /\left\{0^{\circ}\right\}$ (the open interval from $-180^{\circ}$ to $180^{\circ}$, with the value $0^{\circ}$ removed).

An angle or a fold-angle set is fully folded (F) if all angles in the set are in $\left\{F \equiv-180^{\circ}, 180^{\circ}\right\}$. 
Example fold state: PF

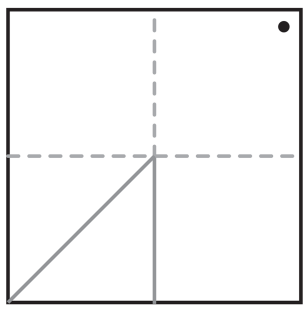

(a)

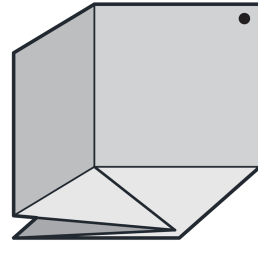

(b)
Figure 3. A crease pattern (a) with fold-angle assignment that will result in a mixed fold state (b). The fold state is a PF fold state with a complete fold-angle set of $\left(90^{\circ}, 90^{\circ}, 90^{\circ},-180^{\circ}, 180^{\circ}\right)$.

An angle or a fold-angle set is flat-folded if all angles in the set are in the set $\left\{-180^{\circ}, 0^{\circ}, 180^{\circ}\right\}$. We do not discuss the flat-folded state further because we categorize the foldangle sets it describes using two more specific fold types. See Fig. 4.

A fold-angle set is mixed-folded if its fold angles are not a single type. For example, the fold state shown in Fig. 3 is a mixed fold state because it has a complete fold-angle set $\left(90^{\circ}, 90^{\circ}, 90^{\circ},-180^{\circ}, 180^{\circ}\right)$, which come from two sets, the $P$ and $F$.

We define the labels for the fold-angle sets based on the minimal sets $(U, P, F)$ that include their folds angles. For example, we label a fold-angle set as $U$ whose angles are in the $U$ set, and UP labels a fold-angle set whose angles are in the $U \cup P$ set. There are three single fold-angle set types: $\mathrm{U}$, $\mathrm{P}$, and F. There are four mixed-fold-angle set types: UP, UF, PF, and UPF. Figure 4 shows the relationship between foldangle sets (capital Roman letters) and lists the minimal sets they include (italics and set notation).

A fold state is one of these types if its complete fold-angle set is that type. For example, a fold state with a complete fold-angle set of $\left(-180^{\circ}, 180^{\circ}, 180^{\circ}, 180^{\circ}\right)$ is fully folded (F). We label fold states with the same label as their complete fold-angle set.

\section{Functions and fold states of origami-based devices}

The value of fold states in origami-based design stems from the fact that fold states of a type share properties and behaviors characteristic of that type. Designers can then generalize those properties as they evaluate or design patterns for an application. The properties and behaviors of the fold state of a device determine, in part, how well the device performs the various functions of the application. This results in correlations between fold states of a device and functions it performed. We analyzed these correlations exhibited in a large number origami-based device.

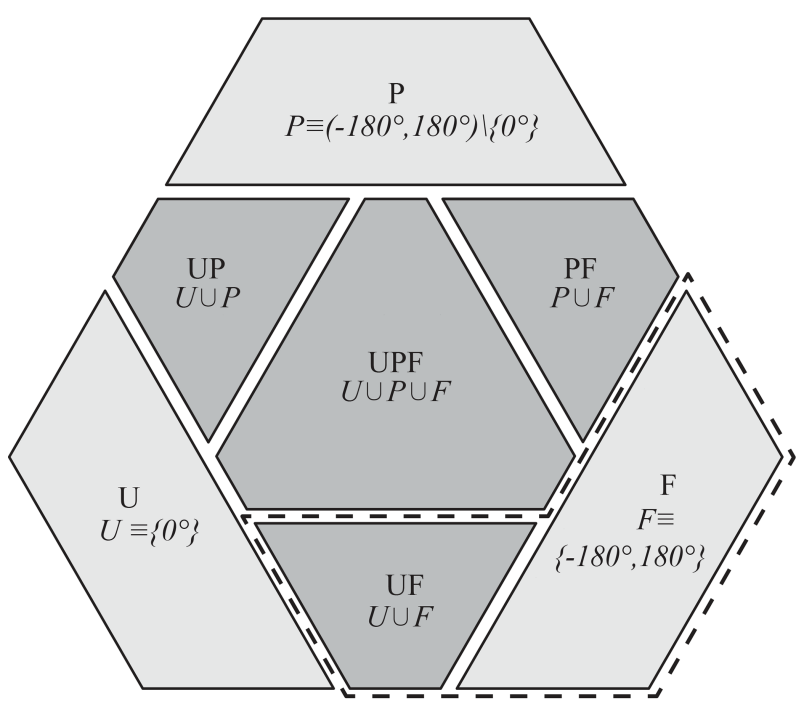

$$
\begin{aligned}
& \text { U - unfolded } \\
& \text { P - partially folded } \\
& \text { F - fully folded } \\
& \text { UP - UP mixed folded } \\
& \text { UF - UF mixed folded } \\
& \text { PF - PF mixed folded } \\
& \text { UPF - UPF mixed folded }
\end{aligned}
$$

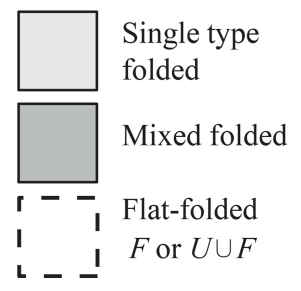

Figure 4. A graphical representation of how the fold-angle space is divided into sets (set notation and italic letters) and their corresponding fold-angle sets (capital Roman letters). The light gray regions are single type folded, the dark gray regions are mixed folded, and the two regions (F and UF) grouped using the dotted line are flat-folded.

The devices analyzed in this review come from reviews of devices and origami principles (Morgan et al., 2017; Morris et al., 2016; Cafarelli et al., 2013; Brownell, 2006; Thrall and Quaglia, 2014) and individual sources including:

Research (Liu et al., 2014; Cheng et al., 2013; Butler et al., 2016; Edmondson et al., 2013; Yang et al., 2017; Miyashita et al., 2015; Felton et al., 2014a; Miura and Natori, 1985; Suzanne Casement, 2012; Kuribayashi et al., 2006; Morgan et al., 2016; Lee et al., 2013; Early et al., 2003; Seymour et al., 2018),

Commercial products (Wee, 2014; Puj LLC, 2018; Fozzils, 2018; Minimum, 2018; Onak, 2018; Bachrach, 2015; Staff, 2018; Plastics Inc., 2018; DesignSwan, 2011; Kraüti, 2018; Junkie, 2018; DesignSwan, 2013; Smith et al., 2017; Parker, 2018; Nellianna, 2018; Fubar, 2011a; Designboom, 2012; Hussey, 2014; Suzanne Casement, 2012; Schielke, 2014; Ihnatko, 2013; Swanner, 2015; Pipetto, 2018; Yang, 2017; bltd, 2010; Hugue, 2017),

Miscellaneous sources (Thün et al., 2012; Simpson and Elkins, 2018; Griffiths, 2015; Elliott, 2013; Fubar, 2011b; Rehn, 2011; Yong, 2013; Felton et al., 2014b). 
Table 1. Examples of origami-based device for each fold state. Bowl (Fozzils ${ }^{\mathrm{TM}}$ ) (Fozzils, 2018), Forcpdf(Morgan et al., 2017), Colander (B\&R Plastics Inc ${ }^{\mathrm{TM}}$ ) (Plastics Inc., 2018), Glasses Case (Warby Parker ${ }^{\mathrm{TM}}$ ) (Parker, 2018), Camping Pot (Bear Minimum ${ }^{\mathrm{TM}}$ ) $($ Minimum, 2018), Solar Array (Zirbel et al., 2013), and Tablet Case (Pipetto ${ }^{\text {TM }}$ ) (Pipetto, 2018). The hashes on the crease pattern indicate where facets are connected. All figures are used with permission by their respective owners.
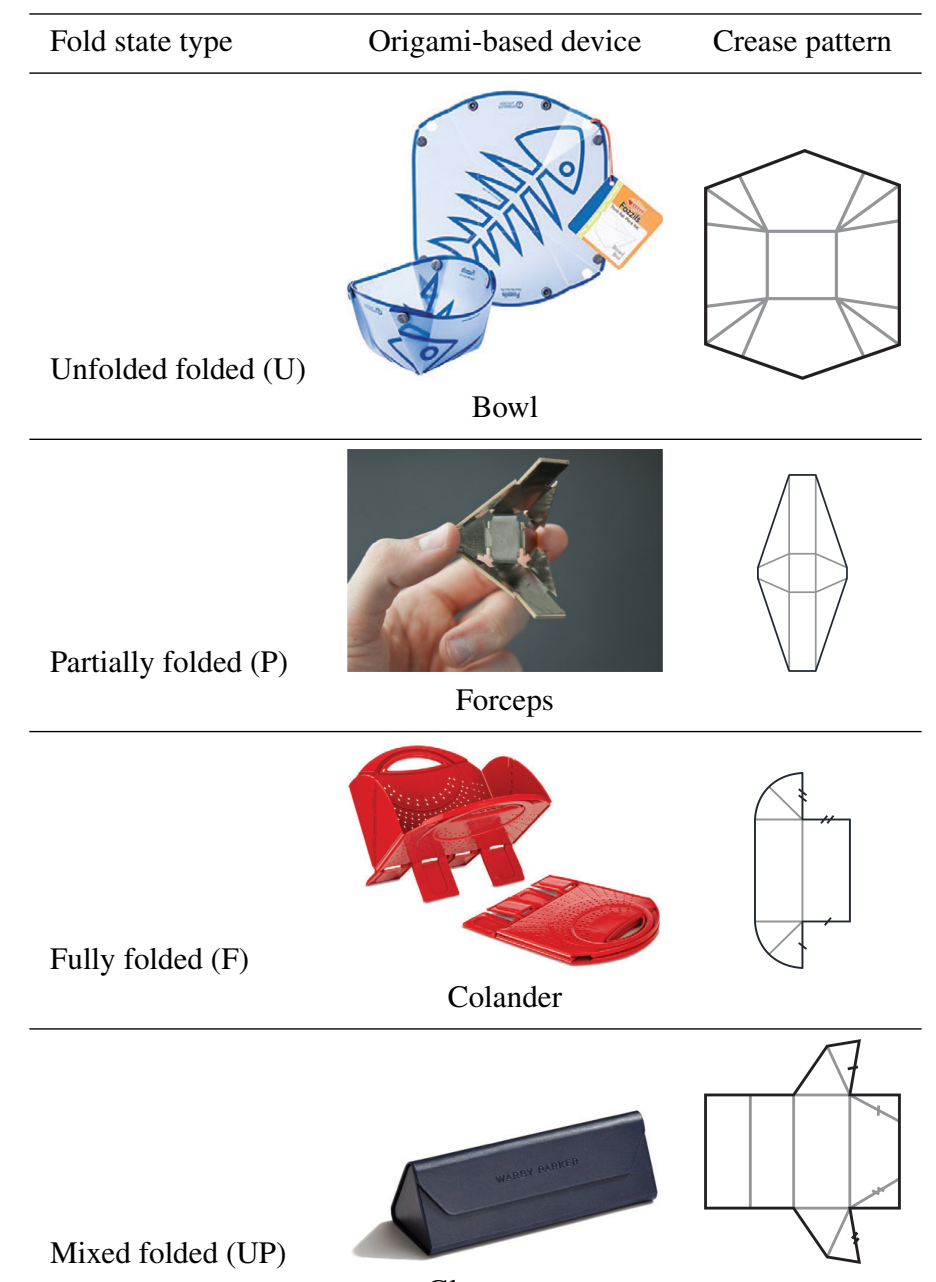

Glasses case

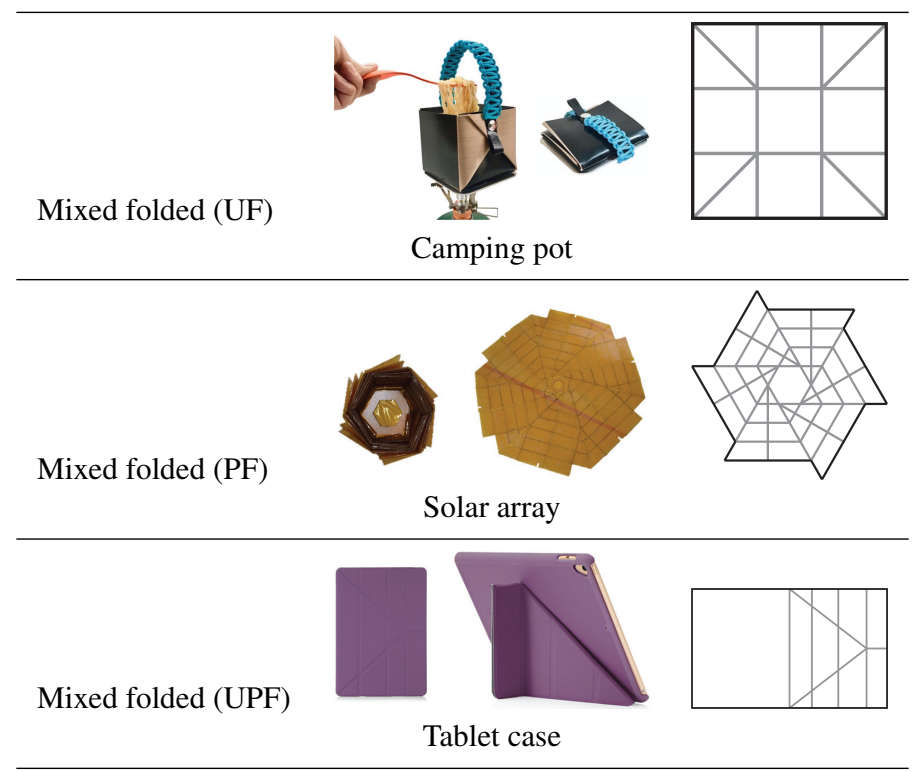


Table 2. A list of the origami-based devices used in Tables 3 and 4, their device number and reference number.

\begin{tabular}{|c|c|c|c|c|c|}
\hline $\begin{array}{l}\text { Device } \\
\text { number }\end{array}$ & $\begin{array}{l}\text { Product } \\
\text { description }\end{array}$ & Reference & $\begin{array}{l}\text { Device } \\
\text { number }\end{array}$ & $\begin{array}{l}\text { Product } \\
\text { description }\end{array}$ & Reference \\
\hline 1 & Antenna & Liu et al. (2014) & 36 & Kiosk & Morris et al. (2016) \\
\hline 2 & Backpack & Morgan et al. (2017) & 37 & Lamp & Cafarelli et al. (2013) \\
\hline 3 & Baffling & Thün et al. (2012) & 38 & Lampshade & Nellianna (2018) \\
\hline 4 & Bag & Wee (2014) & 39 & Phone & Fubar (2011b) \\
\hline 5 & Ballistic Barrier & Seymour et al. (2018) & 40 & Planter & Morris et al. (2016) \\
\hline 6 & Bath Tub & Puj LLC (2018) & 41 & Ring Box & Fubar (2011a) \\
\hline 7 & Bath Tub & Puj LLC (2018) & 42 & Robot & Miyashita et al. (2015) \\
\hline 8 & Battery & Cheng et al. (2013) & 43 & Robot & Felton et al. (2014a) \\
\hline 9 & Bellows & Butler et al. (2016) & 44 & Shelter & Designboom (2012) \\
\hline 10 & Blanket & Morris et al. (2016) & 45 & Shelter & Cafarelli et al. (2013) \\
\hline 11 & Boat & Simpson and Elkins (2018) & 46 & Shelter & Thrall and Quaglia (2014) \\
\hline 12 & Boat & Griffiths (2015) & 47 & Shroud & Morgan et al. (2017) \\
\hline 13 & Boat & Cafarelli et al. (2013) & 48 & Sofa & Hussey (2014) \\
\hline 14 & Boat & Cafarelli et al. (2013) & 49 & Sofa & Rehn (2011) \\
\hline 15 & Boots & Morris et al. (2016) & 50 & Solar Array & Miura and Natori (1985) \\
\hline 16 & Bowl & Fozzils (2018) & 51 & Solar Array & Zirbel et al. (2013) \\
\hline 17 & Camping Pot & Minimum (2018) & 52 & Speaker & Cafarelli et al. (2013) \\
\hline 18 & Canoe & Onak (2018) & 53 & Spoon & Cafarelli et al. (2013) \\
\hline 19 & Chair & Brownell (2006) & 54 & StarShade & Suzanne Casement (2012) \\
\hline 20 & Chair & Elliott (2013) & 55 & SunShade & Schielke (2014) \\
\hline 21 & Chair & Bachrach (2015) & 56 & Stent & Kuribayashi et al. (2006) \\
\hline 22 & Chair & Staff $(2018)$ & 57 & Stool & Cafarelli et al. (2013) \\
\hline 23 & Colander & DesignSwan (2011) & 58 & Stool & Yong (2013) \\
\hline 24 & Colander & Plastics Inc. (2018) & 59 & Table & Cafarelli et al. (2013) \\
\hline 25 & Cup & Cafarelli et al. (2013) & 60 & Table & Morgan et al. (2016) \\
\hline 26 & Curtain & Kraüti (2018) & 61 & Tablet Case & Ihnatko (2013) \\
\hline 27 & Cutting Board & Junkie (2018) & 62 & Tablet Case & Swanner (2015) \\
\hline 28 & Cutting Board & DesignSwan (2013) & 63 & Tablet Case & Pipetto (2018) \\
\hline 29 & Fairing & Smith et al. (2017) & 64 & Telescope Lens & Early et al. (2003) \\
\hline 30 & Forcpdf & Edmondson et al. (2013) & 65 & Utensils & Yang (2017) \\
\hline 31 & Glasses Case & Parker (2018) & 66 & Waste Bin & bltd (2010) \\
\hline 32 & Green House & Cafarelli et al. (2013) & 67 & Wheel & Felton et al. (2014b) \\
\hline 33 & Helmet & Yang et al. (2017) & 68 & Wheel & Lee et al. (2013) \\
\hline 34 & Ice Bucket & Morris et al. (2016) & 69 & Wine Tote & Hugue (2017) \\
\hline 35 & Kayak & Morris et al. (2016) & & & \\
\hline
\end{tabular}

\subsection{Device criteria}

Devices in this study have at least one interior vertex (to exclude simple fan-folding devices), revolute-like joints and at least two different fold states (to exclude devices that look like origami but do not fold like origami, such as a cast ceramic figure). Table 1 gives an example of an origami-based device for each fold-state type. The table also shows the crease patterns for each device. Table 2 gives a complete list of the 69 origami-based devices in this study.

\subsection{Function criteria}

Functions are divided into two groups: (1) those performed by the device (Table 4) and (2) those performed on the device (Table 3). For example, the origami blanket insulates a user; the function is performed by the blanket (Morris et al., 2016).
A worker assembles the fairing, the function is performed on the truck fairing (Smith et al., 2017).

Functions are labeled in Tables 3 and 4 using the functional basis developed by Hirtz et al. (Hirtz et al., 2002).

\subsection{Fold state criteria}

Fold state(s) for each function were determined using images or folding the crease pattern. Only folds connected to an interior vertex were used to determine the fold state of the device. This is to avoid including simple flaps in the fold state. We list the device number in rows of the functions performed on or by the device. The column(s) correspond with the fold state(s) of the device. 
Table 3. We list each device's number in rows corresponding with the functions performed on the device and columns of its corresponding fold states. See Table 2 for device numbers and references. Functions are labeled using the functional basis developed by Hirtz et al. (2002).

\begin{tabular}{|c|c|c|c|c|c|c|c|}
\hline $\begin{array}{l}\text { Functions on the } \\
\text { device }\end{array}$ & $\mathrm{U}$ & $\mathrm{P}$ & $\mathrm{F}$ & UP & UF & $\mathrm{PF}$ & UPF \\
\hline $\begin{array}{l}\text { Manufactured by } \\
\text { dividing material }\end{array}$ & $\begin{array}{l}1,8,9,11,12,13,16,17,18 \\
21,22,25,32,35,38,39,41 \\
47,51,64,65,66,67\end{array}$ & - & - & - & - & - & - \\
\hline $\begin{array}{l}\text { Manufactured by } \\
\text { joining material }\end{array}$ & $\begin{array}{l}1,2,5,8,10,17,39,42,48 \\
52,55,56,59,60,61,65\end{array}$ & $\begin{array}{l}1,2,9 \\
36,66 \\
67\end{array}$ & - & 14,49 & 12 & 33 & - \\
\hline $\begin{array}{l}\text { Manufactured by } \\
\text { removing material }\end{array}$ & 43 & - & - & - & - & - & - \\
\hline Stored & $\begin{array}{l}4,6,11,16,19,20,23,27,28 \\
39,43,48,63\end{array}$ & - & $\begin{array}{l}1,5,24,29, \\
31,36,41,44, \\
45,46,47,57, \\
58,67\end{array}$ & 18 & $\begin{array}{l}7,11,13 \\
14,17,33 \\
34,35\end{array}$ & 25,40 & 62 \\
\hline Transported & $4,11,16,20,43$ & - & $\begin{array}{l}5,44,45 \\
46,50\end{array}$ & $\begin{array}{l}2,4 \\
18,30\end{array}$ & $\begin{array}{l}7,11,13 \\
14,17,35 \\
55,56\end{array}$ & 52,54 & - \\
\hline
\end{tabular}

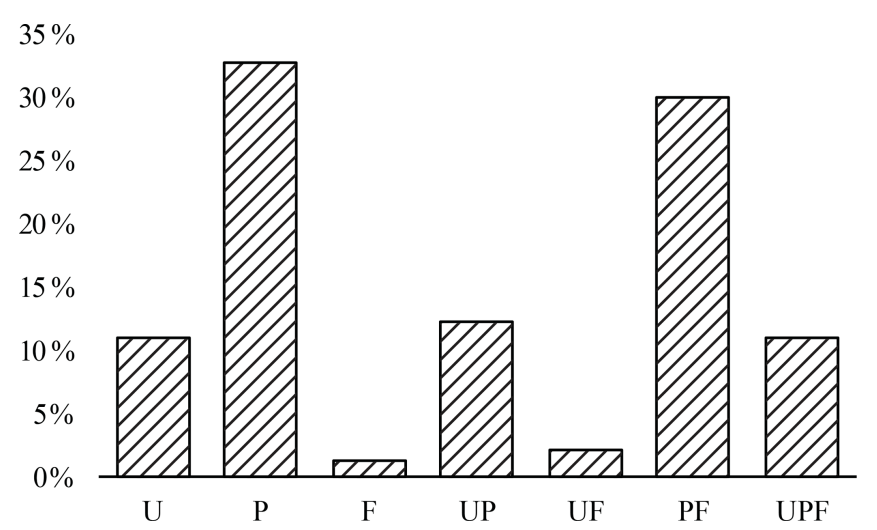

Figure 5. The frequency (as a percentage) of the device fold states when performing a function.

\subsection{Correlations between device fold states and their functions}

Given the emphasis on flat-foldable origami, Tables 3 and 4 illustrate some unexpected results. P and PF are the most common fold states and $\mathrm{F}$ and $\mathrm{UF}$ are the least common when a device performs a function (see Fig. 5). The frequent use of the $\mathrm{P}$ and PF states suggests that additional emphasis should be placed on researching ways to create and maintain $\mathrm{P}$ and PF states.

$\mathrm{U}, \mathrm{F}$ and UF fold states are common states for storage and transportation (see Fig. 6). This is not surprising because these states often have high spatial densities.

We only listed fold states that were clearly used for manufacturing in Table 3. For example, devices manufactured

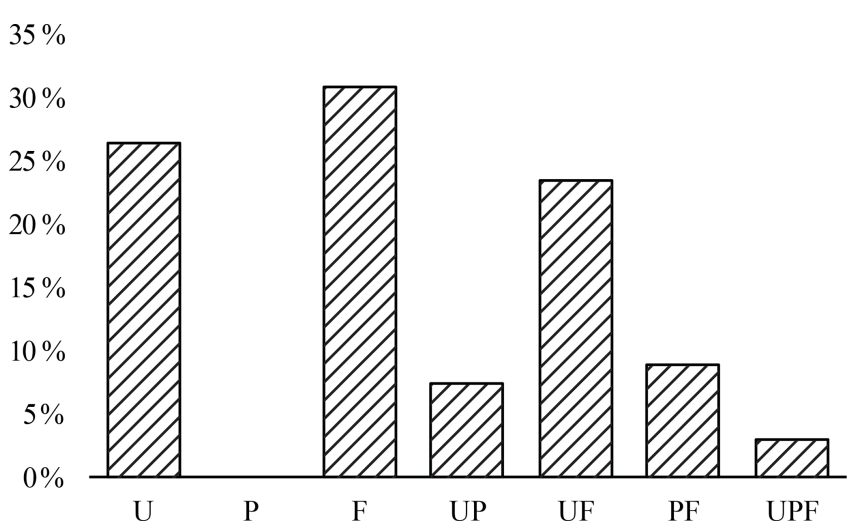

Figure 6. The frequency (as a percentage) of the device fold states when the device is transported or stored.

from a planar sheet, such as the camping pot (Minimum, 2018), require at least one manufacturing step in the unfolded state. Devices assembled from multiple individual parts, such as the fairing, do not have a clear manufacturing fold state. Of the fold states recorded for manufacturing, $80 \%$ were the unfolded state (see Fig. 7). This percentage is probably inappropriately amplified because manufacturing in the unfolded state is easy to positively identify. Nevertheless, the majority of the origami-based devices in this study are manufactured using at least one continuous sheet. 
Table 4. We list each device's number in rows corresponding with the functions performed by the device and columns of its corresponding fold states. See Table 2 for device numbers and references. Functions are labeled using the functional basis developed by Hirtz et al. (2002).

\begin{tabular}{|c|c|c|c|c|c|c|c|}
\hline Functions by the Device & $\mathrm{U}$ & $\mathrm{P}$ & $\mathrm{F}$ & UP & UF & $\mathrm{PF}$ & UPF \\
\hline Change mechanical force & - & 30 & - & - & - & - & - \\
\hline Channel liquid & _- & - & - & 56 & - & - & - \\
\hline $\begin{array}{l}\text { Collect electormagnetic } \\
\text { energy }\end{array}$ & 50,51 & - & - & - & - & - & - \\
\hline Contain material & - & $\begin{array}{l}2,7,30,40 \\
42,43\end{array}$ & - & $2,4,40$ & - & $\begin{array}{l}6,16,17 \\
25,27,28 \\
40,53,66\end{array}$ & 34,65 \\
\hline $\begin{array}{l}\text { Convert magneticomotive } \\
\text { force }\end{array}$ & - & 42 & - & - & - & - & - \\
\hline $\begin{array}{l}\text { Convert rotational angular } \\
\text { velocity }\end{array}$ & - & 43 & - & - & - & - & - \\
\hline $\begin{array}{l}\text { Decrement electromagnetic } \\
\text { intensity }\end{array}$ & $26,54,55$ & - & - & - & - & 38 & 26 \\
\hline $\begin{array}{l}\text { Decrement pneumatic } \\
\text { pressure }\end{array}$ & - & - & - & 29 & - & - & - \\
\hline $\begin{array}{l}\text { Distribute electromagnetic } \\
\text { intensity }\end{array}$ & 26 & - & - & - & - & 38 & 26 \\
\hline Export material & $\begin{array}{l}6,16,17 \\
23,30,65\end{array}$ & 7 & - & 24 & - & - & - \\
\hline Extract liquid & - & - & - & 24 & - & $23,27,28$ & _- \\
\hline Function & - & - & - & - & - & - & - \\
\hline $\begin{array}{l}\text { Import electromagnetic } \\
\text { intensity }\end{array}$ & - & - & - & - & - & 55 & - \\
\hline Import material & - & 45 & 36 & - & - & - & - \\
\hline Increment acoustic pressure & - & - & - & 52 & - & - & - \\
\hline $\begin{array}{l}\text { Increment electromagnetic } \\
\text { intensity }\end{array}$ & 64 & 1 & - & - & - & - & - \\
\hline Inhibit liquid & - & 11 & - & 14 & - & 11,12 & $13,18,35$ \\
\hline Inhibit material & 27,28 & $\begin{array}{l}2,5,9,15 \\
36,45,46 \\
47\end{array}$ & - & $2,31,44$ & - & - & - \\
\hline Inhibit mechanical force & 61 & $5,7,69$ & - & 4,31 & 62,63 & 6,33 & - \\
\hline Inhibit thermal heat flow & 10,48 & $7,32,69$ & - & - & - & 6 & 34 \\
\hline Measure material & - & - & - & _- & - & - & 65 \\
\hline Position material & 43,64 & $\begin{array}{l}1,37,39 \\
41,43,68\end{array}$ & - & 52,63 & - & 61 & 62,63 \\
\hline Regulate acoustic pressure & 3 & - & - & - & - & 3 & - \\
\hline $\begin{array}{l}\text { Regulate electromagnetic } \\
\text { intensity }\end{array}$ & - & 1 & - & - & - & - & - \\
\hline Regulate mechanical torque & _- & 67 & - & _- & _- & _- & _- \\
\hline Rotate & - & 9 & - & - & - & 9 & - \\
\hline Secure material & - & 15 & - & - & - & 33 & - \\
\hline Stabilize material & - & 5,20 & - & 49 & - & $\begin{array}{l}19,21,22, \\
48\end{array}$ & - \\
\hline Store electrical energy & _- & _- & 8 & _- & - & - & _- \\
\hline Support material & 59 & $60,67,68$ & - & 56,63 & - & 61,65 & 62,63 \\
\hline Support mechanical force & - & $\begin{array}{l}11,20,45 \\
46,58\end{array}$ & - & $14,44,49$ & - & $\begin{array}{l}11,12,19, \\
21,22,48, \\
57\end{array}$ & $13,18,35$ \\
\hline Translate material & - & 42,43 & - & - & - & - & - \\
\hline $\begin{array}{l}\text { Transmit electromagnetic } \\
\text { intensity }\end{array}$ & - & 32 & - & - & - & - & - \\
\hline Transmit mechanical force & - & $30,67,68$ & - & - & - & - & - \\
\hline Transport material & - & 11,69 & - & 14 & - & $11,12,53$ & $13,18,35$ \\
\hline
\end{tabular}




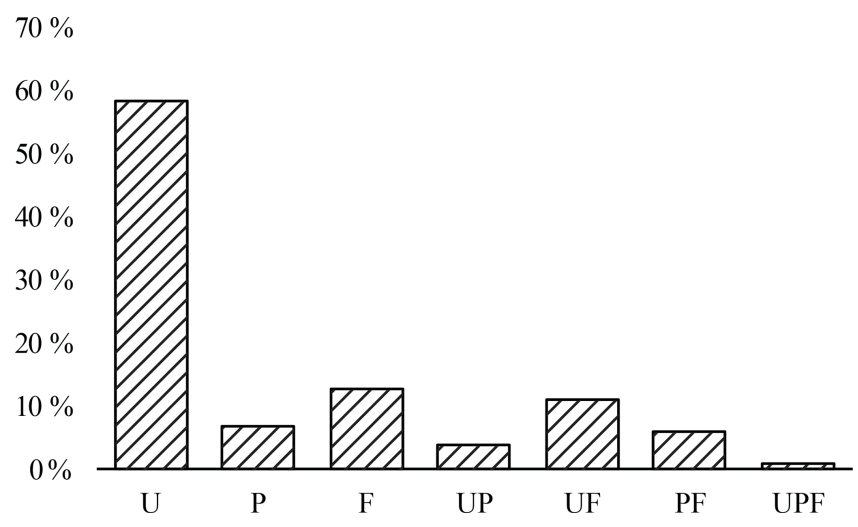

Figure 7. The frequency (as a percentage) of the device fold states when the device is manufactured. These values only represent devices with clear manufacturing fold states.

$$
\begin{aligned}
& \text { Fold angle } \\
& \text { assignment } \\
& u \quad----p \quad f
\end{aligned}
$$

Figure 8. Legend giving the fold angles assignment of the example fold states.

\section{Fold state properties and behaviors}

In this section we discuss some common properties and behaviors of each fold-state type, as well as the functions performed by devices in these fold states. We will assume that the origami patterns are rigidly foldable (Tachi, 2010). Thick-panel origami is not discussed.

For each fold state, there is figure containing a crease pattern on the left (panel a) and a corresponding fold state on the right (panel b). In these figures the dashed, solid, and dotted lines respectively represent unfolded, partially folded, and fully folded folds, as seen in Fig. 8.

\subsection{Unfolded state (U)}

Unfolded states are essentially planar surfaces with designated crease lines. However, the unfolded state is important for several reasons: (1) the majority of origami-based devices are manufactured in the unfolded state from sheetlike materials, (2) unfolded states are bifurcation points in the origami's path of motion, (3) most crease patterns are a 2-D embedding of the unfolded state, as seen in Fig. 9.

All the creases in the unfolded state are coplanar, which creates the bifurcation point in the fold motion, as seen in Fig. 10 (Tachi and Hull, 2017; Waitukaitis et al., 2015). In a degree-four vertex in the unfolded state the minor and major folds form simultaneously; this is not necessarily true for the UP, UF, or UPF states. When origami bifurcates, the fold order changes, forming new fold states with different properties (Waitukaitis et al., 2015).
Example fold state: $\mathrm{U}$

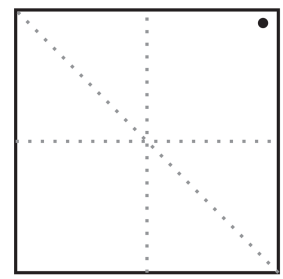

(a)

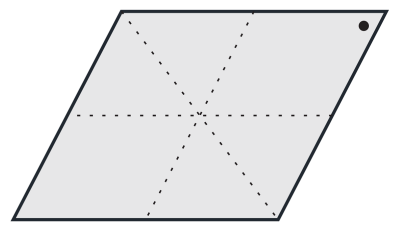

(b)
Figure 9. A crease pattern (a) with fold-angle assignment that will result in an unfolded fold state (b). The legend for fold-angle assignment is given in Fig. 8. Although the crease pattern appear the same as the fold state, the crease pattern is a planar embedding of the fold state.

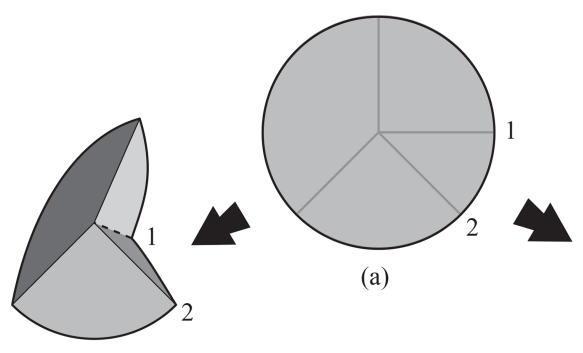

(b)

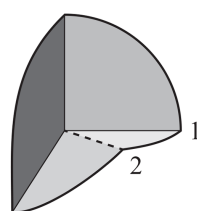

(c)
Figure 10. Crease patterns in the unfolded state (a) can bifurcate so that either fold 1 in fold state (b) or 2 in fold state (c) (represented by the dotted line) has a fold angle with an opposite sign to the other fold angles.

Unfolded states are often used for storage and transportation because they have low thickness and high spatial density. However, they also typically have large dimensions. The unfolded state is especially common for devices that are transported right after being manufactured in the unfolded state.

Unfolded states have the largest projected area for a given origami pattern, which is one reason why it has been used for the space telescope, flasher solar array, and starshade (Early et al., 2003; Zirbel et al., 2013; Lo, 2009).

\subsection{Partially folded state (P)}

For a given crease pattern there are a finite number of fully folded and unfolded states, which means that those states are especially useful for communicating information about a fold state. On the other hand, there is a certain amount of ambiguity communicated with the term partially folded state. This is because unlike the unfolded and fully folded states, the partially folded is not based on a finite set of fold angles.

A partially folded state is guaranteed to have a non-planar shape because the facets of the origami are not coplanar. As well, the partially folded set $P$ is the basis for three mixed fold states, which are potentially finite for a given origami pattern. 
Example fold state: $\mathrm{P}$

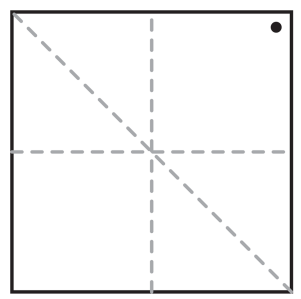

(a)

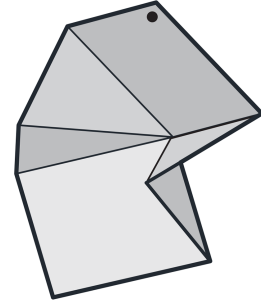

(b)
Figure 11. A crease pattern (a) with fold-angle assignment that will result in a partially fold state (b). The legend for fold-angle assignment is given in Fig. 8. The partially folded state is one of the most common fold states for performing a function. The fold state (b) shown in Fig. 9 has the same crease pattern, demonstrating how a single crease pattern can have multiple fold states.

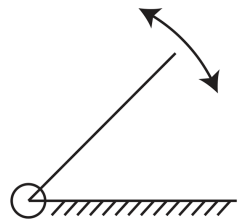

(a) Two directions of motion

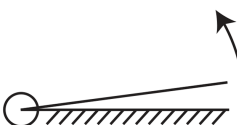

(b) One direction of motion
Figure 12. A crease can fold in two directions (a) until it reaches the fully folded state (b). In the fully folded state the motion of one facet is limited by intersection with the other facet. An external constraint in the opposite direction fully constrains the crease (c).

The partially folded state is the most common fold state for devices performing a function (see Fig. 5). This is because devices often perform functions that interact with our 3 -D world, not just in a plane. One example is loading conditions. The ballistic barrier is deployed to the partially folded state with an out-of-plane base to prevent tipping (Seymour et al., 2018). The partially folded state also provides higher stiffness in bending than the unfolded state.

\subsection{Fully folded state (F)}

The fully folded state is distinct from other fold states because each pair of adjacent facets is coplanar and they intersect.

In a zero thickness model, the facets of the fully folded state intersect, creating a hard stop in one fold direction. By constraining the crease in the opposite fold direction the crease is completely constrained, as seen in Fig. 12. When the loading on a fully folded crease results in a moment in the constrained direction, additional constraints may not be necessary. This loading situation is an elegant way to create a stable fold state without adding hardware.

Designers often chose the fully folded state for storing or transportation of a device, because it typically has high spa-

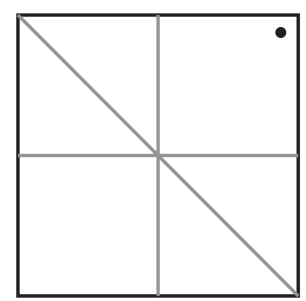

(a)

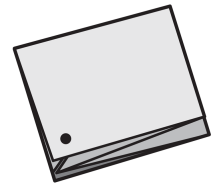

(b)
Figure 13. A crease pattern (a) with fold-angle assignment that will result in a fully fold state (b). The legend for fold-angle assignment is given in Fig. 8. The fully folded state is often used for transportation or storage, as seen in Fig. 6.

tial density and small dimensions relative to other states of the same origami pattern.

Only origami patterns that are flat-foldable have fully folded states.

\subsection{Mixed fold state}

Mixed fold states (UF, UP, PF, or UPF) are the groups of fold states that have fold angles from more than one set of fold angle values $(U, P, F)$. Mixed fold states combine the properties and behaviors that come from having $\mathrm{U}, \mathrm{P}$, and $\mathrm{F}$ fold angles. For example, the UP, PF, and UPF fold states all have $P$ fold angles and are non-planar fold states.

We discuss the mixed fold states that have unfolded fold angles (UP, UF, and UPF) as a group, because they share some similar properties and behaviors. Each mixed fold state is then discussed individually.

\subsubsection{Unfolded creases in mixed fold states}

The UP, UF, and UPF all contain at least one unfolded fold angle. These states are interesting because the unfolded state on first inspection appears to add no contribution to the overall shape of the fold state. The fold state would be the same shape if the two facets adjacent to the unfolded crease were combined into one facet, by removing the unfolded crease. For example, the fold state in Fig. 11 is the same shape as in Fig. 14. However, there are some reasons why an unfolded fold angle is included in a mixed fold state. For example:

1. To make an origami pattern rigidly foldable. For example, the metal bag designed by Wu and You (2011).

2. To allow origami with the same overall shape additional forms of motion. For example, the fold state in Figs. 21 and 19 have the same shape, but only the fold state in Fig. 21 is flat-foldable.

3. As a biproduct of the fabrication process. For example, pre-creasing in traditional paper folding. 


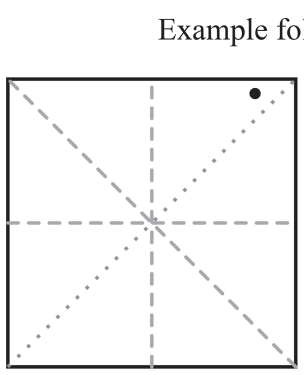

(a)

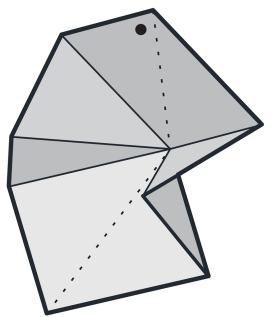

(b)
Figure 14. A crease pattern (a) with fold-angle assignment that will result in a UP fold state (b). The legend for fold-angle assignment is given in Fig. 8. The UP fold state is often used in devices that conform to the material they contain or support. It also occurs in devices with large cavities, especially those that are non-developable.

4. If the fold state is reconfigurable and has inactive creases, such as the superimposed origami patterns by Liu et al. (2016).

\subsubsection{UP mixed fold state (UP)}

The UP fold state occurs frequently in devices based on origami tessellations with many facets and degrees of freedom, such as the curtain, backpack, sofa, and bag (Kraüti, 2018; Morgan et al., 2017; Rehn, 2011; Wee, 2014). These devices are made of soft materials and are designed to conform to user input.

Devices with the UP fold state often are designed to have large cavities, such as the stent, shelter, planter, and tablet case. These devices maintain their shape because of global interference rather than the local interference of a fully folded crease.

Another common occurrence of the UP fold state is when the device forms a cavity and is non-developable, such as the colander, speaker, fairing, or glasses case (Plastics Inc., 2018; Cafarelli et al., 2013; Smith et al., 2017; Parker, 2018). The unfolded creases allow the devices to fold flat.

Sometimes the unfolded creases are prevented from folding by the partially folded creases and can only fold in the fully folded or unfolded state. This is illustrated in Fig. 15 and is used by Liu et al. (2016).

\subsubsection{UF mixed fold state (UF)}

The UF fold state is typically used for storage (Fig. 6) in two scenarios. (1) When a device is folded along one pattern to perform its main function and folded along another superimposed pattern for storage, such as the kayak and boats (Morris et al., 2016; Cafarelli et al., 2013). (2) When a device is non-developable, such as the bath tub, ice bucket, and helmet (Puj LLC, 2018; Morris et al., 2016; Yang et al., 2017).

All origami that folds sequentially passes through either the UF or UPF mixed fold states. This is because these states

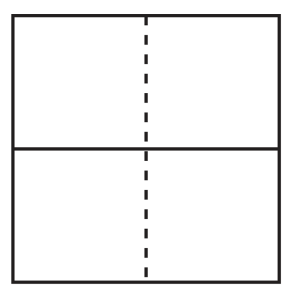

(a)

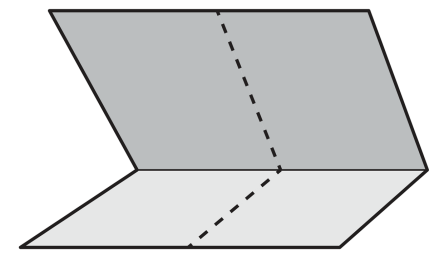

(b)
Figure 15. The UP state (b) based on the crease pattern (a), cannot fold along the creases represented by the dotted line because the creases are not collinear.

Example fold state: UF

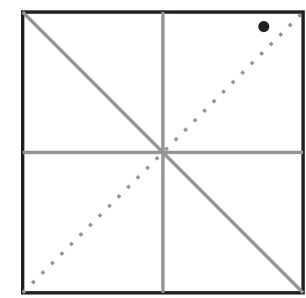

(a)

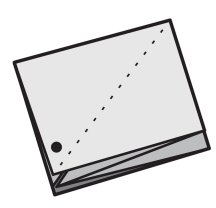

(b)
Figure 16. A crease pattern (a) with fold-angle assignment that will result in a UF fold state (b). The legend for fold-angle assignment is given in Fig. 8. The UF state can gain added degrees of freedom if the unfolded creases are collinear. For example, the fold state could fold along the dotted line.

acquire an additional path of motion when two unfolded creases become collinear (assuming that the rest of the pattern allows folding). For example, see Figs. 16 and 17.

\subsubsection{PF mixed fold state (PF)}

The PF state is often used to create three dimensional structures, because the PF states share similarities with both fully folded states and partially folded states. The partially folded fold angles ensure a three dimensional configuration and the fully folded fold angles decrease the degrees of freedom in a fold direction. See Fig. 19.

A single degree of freedom origami pattern has a PF fold state if it is not flat-foldable.

The fully folded fold angles results in small dimensions and high spatial density locally, but the partially folded fold angles ensure that the overall shape is non-planar. The combination of these fold angles makes this state a strong candidate for storage or transportation if the device will be stowed in a three dimensional shape, such as the flasher solar array (Zirbel et al., 2013) or the origami planter (Morris et al., 2016).

Including fully folded fold angles in a fold state is an effective method for creating a specific shape. A fold state with two adjacent fully folded angles forms a flap that is copla- 


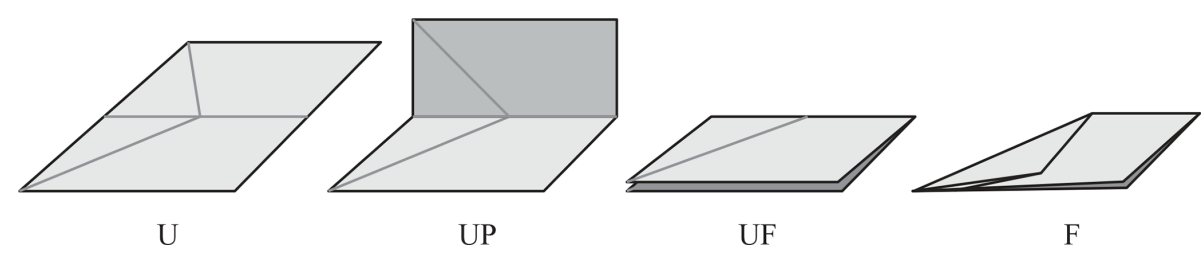

Figure 17. A degree four vertex that is sequentially folded. In the UF state the two diagonal creases are collinear which allows them to fold.
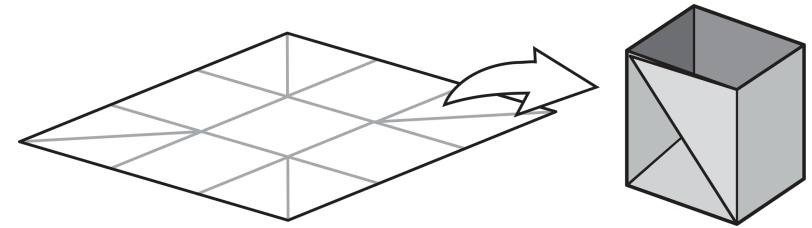

(a)

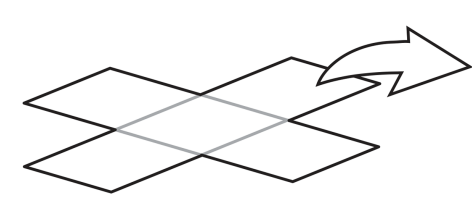

(b)

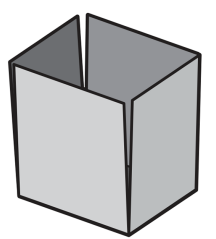

Figure 18. Both fold states shown in (a) and (b) have a similar overall shape when folded, but the fold state in (a) has a water-tight seam because the surface is unbroken.

Example fold state: UPF

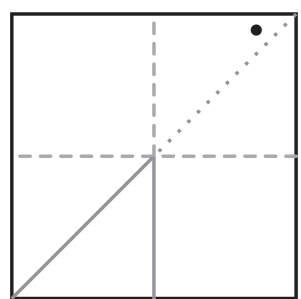

(a)

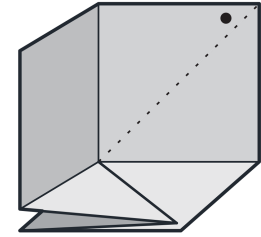

(b)
Figure 19. A crease pattern (a) with fold-angle assignment that will result in a PF fold state (b). The legend for fold-angle assignment is given in Fig. 8. The PF fold state is useful for creating 3-D structures with reduced degrees of freedom.

nar with an adjacent facet. This essentially removes the flap from the overall shape of the origami (as long as the flap is bounded by the facet). The seam formed at the base of the flap remains an unbroken surface, unlike if the flap was actually cut out.

The camping pot in Table 1 is an example of where this can be useful. The corner facets are fully folded making them coplanar with facets that form the side walls of the pot. This "removes" the corner pieces from the geometry to create the box shape but the seams are still water-tight (see Fig. 18).

\subsubsection{UPF mixed fold state (UPF)}

The UPF state occurs frequently when considering the complete fold-angle set of the entire device; however, it seldom occurs in a single vertex. Of the eight devices that have a UPF fold state, only two have a vertex with unfolded, partially folded, and fully folded fold angles.

The tablet case in Table 1 is an excellent example of how three types of fold angles are combined to result in a fold state with specific properties and behaviors. The tablet case has unfolded fold angles because it is reconfigurable, partially folded fold angles because it is 3-D, and fully folded fold angles which limits the fold motion to support the device.

\section{Designing devices using fold states}

One of the primary steps for designing an origami-based device is selecting an origami pattern. Fold states do not determine the origami pattern, however, they can be useful in directing a designer towards certain patterns. This is because some patterns are more conducive for achieving certain fold states. For example, the UP fold state typically occurs in nondevelopable origami patterns. Fully-folded states are only achieved by flat-foldable patterns. Mixed fold states with unfolded fold angles typically occur in patterns that are superimposed to be reconfigurable.

Two potential methods of selecting fold state are (1) selecting combination fold-angle types that will result in a fold state with desired properties, (2) using Tables 3 and 4 to find fold states that commonly perform the desired sets of functions.

\subsection{Composing fold states from fold angles}

We typically consider that a fold state defines the facets, creases, and complete fold-angle set. However, for design it is potentially useful to look at it the other way around, that the facets, creases, and complete fold-angle set defines the fold state. This allows a designer to look at each component individually to consider what properties or behavior they contribute to the whole.

As discussed in the previous section, each fold-angle type contributes distinct properties. When designing an origamibased device, an engineer can combine specific fold-angle types to result in a fold state with the those properties. Fig- 


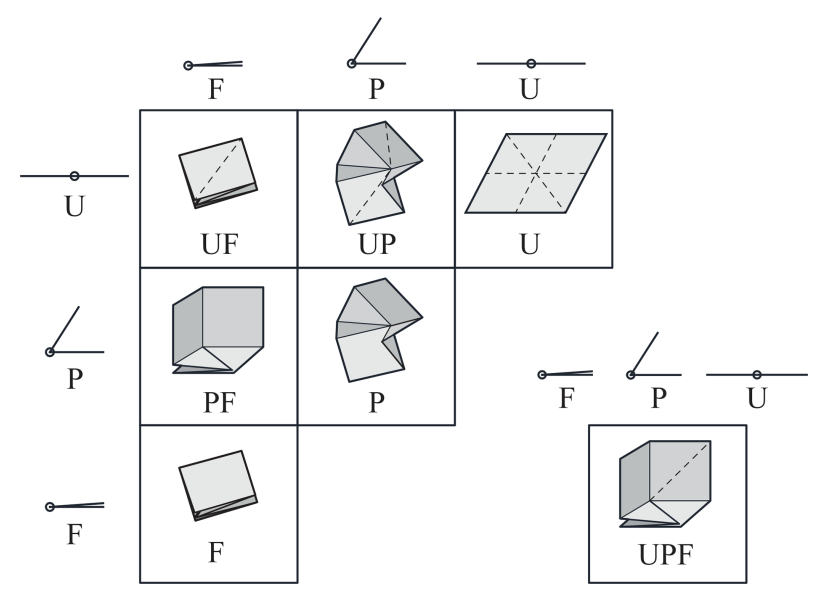

Figure 20. An examples of how fold angles from each sets $(U, P$ and $F$ ) can be combined to form different fold states. Each vertex is an example of one of the seven fold states (larger depictions of the vertices are given with there respective fold state in Sect. 4).

Example fold state: UPF

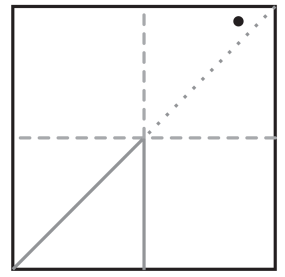

(a)

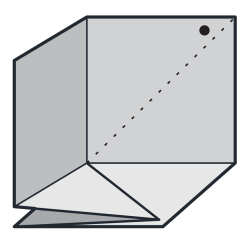

(b)
Figure 21. A crease pattern (a) with fold-angle assignment that will result in a UPF fold state (b). The legend for fold-angle assignment is given in Fig. 8. The UPF state has at least two pairs of coplanar facets, one pair separated by a fully folded crease and another by an unfolded crease.

ure 20 shows an examples of how individual fold angles are combined in the fold state of various vertices.

For example, if we were designing an origami-based chair, we might select partially folded fold angles so the chair has a 3-D shape and fully folded fold angles to limit the chairs fold motion. If the $\mathrm{P}$ and $\mathrm{F}$ fold angles were the only types of fold angles in the complete fold-angle set, the fold state would be a PF fold state. Which indicates that the PF fold state is a potential fold state for the device.

\subsection{Using the tables to select fold states}

By referring to Tables 3 and 4 designers can limit the fold states to consider by looking at which ones are commonly used for a similar function. These fold states are likely to have desirable properties. By identifying fold states commonly used by the functions performed simultaneously, a designer can further limit the number to consider. An example of this process:
1. List device functions.

- Function 1

- Function 2

- Function 3

- Function 4

2. Group functions that the device performs simultaneously.

\begin{tabular}{l|l}
\hline Group 1 & Group 2 \\
\hline Function 1 & Function 2 \\
Function 4 & Function 3 \\
\hline
\end{tabular}

3. Using Tables 3 and 4 , list fold states commonly used for each function.

\begin{tabular}{l|l}
\hline Group 1 & Group 2 \\
\hline Function 1: U, P, F & Function 2: P, UP, PF \\
Function 4: P, F, PF & Function 3: U, F, UF \\
\hline
\end{tabular}

4. Identify fold states that are shared between grouped functions.

\begin{tabular}{l|l}
\hline Group 1 & Group 2 \\
\hline Functions 1 \& 4: P, F & Functions 2 \& 3: None \\
\hline
\end{tabular}

Fold states $\mathrm{P}$ and $\mathrm{F}$ are candidates fold states for performing functions 1 and 4 . The designer could then consider the benefits of each fold state or simply begin investigating origami patterns that can achieve either fold state.

Functions 2 and 3 do not share any fold states in common, the designer has to go back and evaluate which function is the more critical for the device, or decide which function will is less effected by the fold state.

This process could be used for designing the ballistic barrier, see Fig. 1. The barrier needs to: inhibit material (stop bullets), inhibit mechanical force (stop bullets), stabilize material (stand on its own), and be stored. From Table 4 the designer can see that fold states $\mathrm{U}, \mathrm{P}$, and UP have been used for inhibiting material; U, P, UP, and PF for inhibiting mechanical force; and $\mathrm{P}, \mathrm{UP}$, and $\mathrm{PF}$ for stabilizing material; $\mathrm{U}$, F, UP, UF, PF, and UPF for storage.

Since the device will need to perform the first three functions simultaneously, the designer could select only fold states that are shared among all of the functions $-\mathrm{P}$ and UP (see Fig. 22). The designer selects the $P$ fold state because the UP fold state can have additional forms of motion, which would be undesirable.

Tables 3 and 4 can also initiate out-of-the-box thinking by indicating less common fold states for a function. For example, the barrier designer could consider potential advantages that come from using the UF or F fold states. In these states the barrier would have multiple layers, meaning it would provide changeable levels of ballistic protection. However, it is 


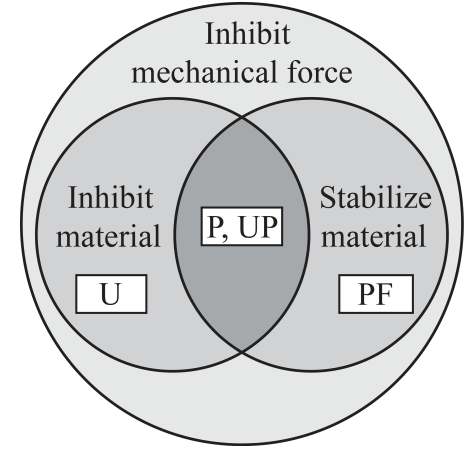

Figure 22. Devices in the P and UP fold states commonly perform the functions: inhibiting material, inhibiting mechanical force, and stabilize material. The Venn diagram shows the overlap of the fold states between these three functions.

also important to consider why these fold states are not currently used to perform a function. For example, a barrier in the UF or F fold states could provide less coverage than the same pattern in another fold state.

\section{Conclusions}

Origami fold states communicate valuable information about the properties and behaviors of origami. The comprehensive list of fold state types proposed categorizes all of the fold states found in origami-based devices. The review of 69 origami-based devices analyzed, support the classification of fold states developed in this paper; there are strong correlations between the devices' fold states and their functions. For example, non-planar fold states are most common for performing functions, while planar fold states are typically used for transportation, storage, and manufacturing.

Fold states for origami-based design can be selected using the properties of individual fold-angle types or by using the correlations established in this paper. We illustrate both methods for using these concepts by selecting a fold state for an origami-based chair and ballistic barrier.

Fold states provide a way to think about and discuss the fold states used in origami-based devices. The properties and behaviors associated with fold states make them a valuable tool in origami-based design.

Data availability. The data used to support the findings of this study are included within the article.

Author contributions. Research was directed by SM and LH. The initial study and concepts were a collaborative effort of AA, SM, RL, and LH. AA lead the writing process. SM contributed by writing and editing throughout the entire work. RB made valuable contributions and edits to the terminology section. LH further refined and edited the manuscript.
Competing interests. The authors declare that they have no conflict of interest.

Acknowledgements. This paper is based on work supported by the National Science Foundation and the Air Force Office of Scientific Research through NSF Grant No. EFRI-ODISSEI-1240417 and the National Science Foundation Graduate Research.

Edited by: Jahangir Rastegar

Reviewed by: two anonymous referees

\section{References}

Bachrach, J.: Computational Design + Fabrication: 2D Design, EECS UC Berkeley, available at: https://inst.eecs. berkeley.edu/ cs194-28/fa15/lectures/2d-design.pdf (last access: 16 May 2018), 2015.

Bern, M. and Hayes, B.: The complexity of flat origami, SODA, 96, 175-183, 1996.

bltd: Waste Bin Kernel Description, available at: http://www.betterlivingthroughdesign.com/accessories/ polywrap-wastepaper-bin/ (last access: 16 May 2018), 2010.

Brownell, B. E.: Transmaterial: A Catalog of Materials, Products and Processes that are Redefining Our Physical Environment, Princeton Architectural Press, 2006.

Butler, J., Morgan, J., Pehrson, N., Tolman, K., Bateman, T., Magleby, S. P., and Howell, L. L.: Highly Compressible Origami Bellows for Harsh Environments, in: ASME 2016 International Design Engineering Technical Conferences and Computers and Information in Engineering Conference, American Society of Mechanical Engineers, V05BT07A001, 2016.

Cafarelli, M., Motta, M., and Storto, M.: Origami: Evoluzione e Ispirazione, thesis, available at: https://issuu.com/mauriziosturt/ docs/tesi_origami_-_evoluzione_e_ispiraz (26 February 2019), 2013.

Cheng, Q., Song, Z., Ma, T., Smith, B. B., Tang, R., Yu, H., Jiang, H., and Chan, C. K.: Folding paper-based lithium-ion batteries for higher areal energy densities, Nano letters, 13, 4969-4974, 2013.

Designboom: Cardboard Shelter Kernel Description, available at: https://www.designboom.com/design/ tricycle-house-and-garden-by-peoples-architecture-office/ (last access: 16 May 2018), 2012.

DesignSwan: Every Origami: 15 Origami Inspired Product Designs, available at: https://www.designswan.com/archives/ every-origami-15-origami-inspired-product-designs.html (last access: 16 May 2018), 2011.

DesignSwan: 8 Cool Multifunctional Cutting Boards, available at: https://www.designswan.com/archives/ 8-cool-multifunctional-cutting-boards.html (last access: 16 May 2018), 2013.

Early, J., Hyde, R., and Baron, R.: Twenty meter space telescope based on diffractive Fresnel lens, Tech. rep., Lawrence Livermore National Lab., CA, USA, 2003.

Edmondson, B. J., Bowen, L. A., Grames, C. L., Magleby, S. P., Howell, L. L., and Bateman, T. C.: Oriceps: Origami-inspired forceps, in: ASME 2013 conference on smart materials, adaptive 
structures and intelligent systems, American Society of Mechanical Engineers, V001T01A027, 2013.

Elliott, A.: Origami Chair Sheet Metal Kernel Description, available at: https://www.youtube.com/watch? v=lgMZsHXJr6w (last access: 16 May 2018), 2013.

Felton, S., Tolley, M., Demaine, E., Rus, D., and Wood, R.: A method for building self-folding machines, Science, 345, 644646, 2014a.

Felton, S. M., Lee, D.-Y., Cho, K.-J., and Wood, R. J.: A passive, origami-inspired, continuously variable transmission, in: 2014 IEEE International Conference on Robotics and Automation (ICRA), 2913-2918, IEEE, 2014b.

Fozzils: Fozzils Ultralight Backpacking Bowl Kernel Description, available at: https://www.fozzils.com/, last access: 16 May 2018.

Fubar, K.: With Clifton Flat Engagement Ring Case, Your Bride-To-Be Would Never See A Proposal Coming, available at: https://mikeshouts.com/ clifton-flat-engagement-ring-case-bride-never-see-proposal-coming/ (last access: 16 May 2018), 2011a.

Fubar, K.: Origami and Phones, Together At Last, available at: https://www.slashgear.com/ origami-and-phones-together-at-last-15146447/ (last access: 16 May 2018), 2011 b.

Greenberg, H. C., Gong, M. L., Magleby, S. P., and Howell, L. L.: Identifying links between origami and compliant mechanisms, Mech. Sci., 2, 217-225, https://doi.org/10.5194/ms-2-217-2011, 2011.

Griffiths, S.: The origami boat a HUMAN can row: Vessel made from a 300ft-long roll of paper carries an adult across a lake, available at: http://www.dailymail.co.uk/sciencetech/ article-2911327.html (last access: 16 May 2018), 2015.

Hirtz, J., Stone, R. B., McAdams, D. A., Szykman, S., and Wood, K. L.: A functional basis for engineering design: reconciling and evolving previous efforts, Res. Eng. Des., 13, 65-82, 2002.

Hugue, M.: Six ways to bring the beauty of origami to your home decor, available at: https://www. theglobeandmail.com/life/home-and-garden/decor/ six-standout-pieces-of-origami-inspired-furniture-and-decor/ article15156918/ (last access: 16 May 2018), 2017.

Hussey, M.: Origami Sofa by Yumi Yoshida unfolds to become a floor mat, available at: https://www.dezeen.com/2014/03/09 (last access: 16 May 2018), 2014.

Ihnatko, A.: Kindle Fire HDX 7-inch review: Third time's the charm, available at: https://www.pcworld.com/article/2051202/ kindle-fire-hdx-7-inch-review-third-times-the-charm.html (last access: 16 May 2018), 2013.

Junkie, G.: Oriboard Origami Cutting Board, available at: http: //www.gadgetify.com/oriboard-origami-cutting-board/, last access: 16 May 2018.

Kraüti, F.: Magnetic Curtain, Kernel Description, available at: http: //www.kraeutli.com/index.php/2008/01/31/magnetic-curtain/, last access: 16 May 2018.

$\mathrm{Ku}$, J. S. and Demaine, E. D.: Folding flat crease patterns with thick materials, J. Mech. Robot., 8, 031003, https://doi.org/10.1115/1.4031954, 2016.

Kuribayashi, K., Tsuchiya, K., You, Z., Tomus, D., Umemoto, M., Ito, T., and Sasaki, M.: Self-deployable origami stent grafts as a biomedical application of Ni-rich TiNi shape memory alloy foil, Mat. Sci. Eng. A-Struct., 419, 131-137, 2006.
Lang, R. J.: Twists, Tilings, and Tessellations: Mathematical Methods for Geometric Origami, AK Peters/CRC Press, 2017.

Lee, D.-Y., Kim, J.-S., Kim, S.-R., Koh, J.-S., and Cho, K.-J.: The deformable wheel robot using magic-ball origami structure, in: ASME 2013 international design engineering technical conferences and computers and information in engineering conference, American Society of Mechanical Engineers, V06BT07A040, 2013.

Lin, J.: A Nomadic Furniture Design for College Students, thesis, available at: http://hdl.handle.net/2142/18533 (last access: 26 February 2019), 2011.

Liu, X., Yao, S., Georgakopoulos, S. V., Cook, B. S., and Tentzeris, M. M.: Reconfigurable helical antenna based on an origami structure for wireless communication system, 2014 IEEE MTT-S International Microwave Symposium (IMS2014), Tampa, FL, USA, 1-6 June 2014, https://doi.org/10.1109/MWSYM.2014.6848553, 2014.

/Liu, X., Gattas, J. M., and Chen, Y.: One-DOF Superimposed Rigid Origami with Multiple States, Sci. Rep., 6, 36883, https://doi.org/10.1038/srep36883, 2016.

Lo, A. S.: Starshade Technology Development, in: astro2010: The Astronomy and Astrophysics Decadal Survey, National Academies of Sciences, Engineering, Medicine, USA, 2009.

Minimum, B.: Bear Bowl, Kernal Description, available at: https: //www.bearminimum.org/, last access: 16 May 2018.

Miura, K. and Natori, M.: 2-D array experiment on board a space flyer unit, Space Power, 5, 345-356, 1985.

Miyashita, S., Guitron, S., Ludersdorfer, M., Sung, C. R., and Rus, D.: An untethered miniature origami robot that self-folds, walks, swims, and degrades, in: 2015 IEEE International Conference on Robotics and Automation (ICRA), 1490-1496, 2015.

Morgan, D. C., Halverson, D. M., Magleby, S. P., Bateman, T. C., and Howell, L. L.: Y Origami?: Explorations in Folding, American Mathematical Society, vol. 104, 2017.

Morgan, M. R., Lang, R. J., Magleby, S. P., and Howell, L. L.: Towards developing product applications of thick origami using the offset panel technique, Mech. Sci., 7, 69-77, https://doi.org/10.5194/ms-7-69-2016, 2016.

Morris, E., McAdams, D. A., and Malak, R.: The State of the Art of Origami-Inspired Products: A Review, in: ASME 2016 International Design Engineering Technical Conferences and Computers and Information in Engineering Conference, American Society of Mechanical Engineers, V05BT07A014, 2016.

Nellianna: Origami Lampshade, Kernel Description, available at: https://www.etsy.com/listing/82568715/ chestnut-paper-origami-lampshade-canary, last access: 16 May 2018.

Onak: Origami Canoe, Kernel Description, available at: http:// onakcanoes.com/, last access: 16 May 2018.

Pagano, A., Yan, T., Chien, B., Wissa, A., and Tawfick, S.: A crawling robot driven by multi-stable origami, Smart Mater. Struct., 26, 094007, https://doi.org/10.1088/1361-665X/aa721e, 2017.

Parker, W.: Warby Parker Glasses Case, Kernel Description, available at: https://i.warbycdn.com/s/f/ 97582daa7f3b79eb0f42d698b1dafa893dea1517?width $=$ 1200\&quality=80, last access: 16 May 2018.

Pipetto: Pipetto Tablet Cases, Kernel Description, available at: https://www.pipetto.co.uk/skin/frontend/default/pipetto/images/ highresimages/iPad/iPad, last access: 16 May 2018. 
Plastics Inc.: Origami Colander, Kernel Description, available at: https://www.brplastics.com/folding-colanders.html, last access: 16 May 2018.

Puj LLC: Bath Tub, Kernel Description, available at: https://puj. com/, last access: 16 May 2018.

Rabinovich, M., Hoffmann, T., and Sorkine-Hornung, O.: Discrete Geodesic Nets for Modeling Developable Surfaces, ACM Transactions on Graphics (TOG), 37, 16, https://doi.org/10.1145/3180494, 2018.

Rehn, A.: Cay Sova, Kernel Description, available at: https://www.youtube.com/watch? $\mathrm{v}=\mathrm{tLgRisKD} 41 \mathrm{w}$ (last access: 16 May 2018), 2011.

Schielke, T.: Light Matters: Mashrabiyas - Translating Tradition into Dynamic Facades, available at: https://www.archdaily.com/ 510226 (last access: 16 May 2018), 2014.

Seymour, K., Burrow, D., Avila, A., Bateman, T., Morgan, D. C., Magleby, S. P., and Howell, L. L.: Origami-Based Deployable Ballistic Barrier, in: Origami 7: The Proceedings from the 7th International Meeting on Origami in Science, Mathematics, and Education, 3, 763-777,, 2018.

Simpson, K. and Elkins, P.: CoroPlast Boat, Kernel Description, available at: https://www.christinedemerchant.com/ boat-styles-coroplast.html, last access: 16 May 2018.

Smith, A. F., Horrell, C. M., Grossmann, J. J., Feldman, J. R., and Bruccoleri, A. R.: Rear-mounted aerodynamic structure for truck cargo bodies, uS Patent 9, 545-960, 2017.

Staff, D.: Origami Style: Paper-Thin, Patio-Ready White Folding Chairs, available at: https://dornob.com/ origami-style-paper-thin-patio-ready-white-folding-chairs/ ?ref=search, last access: 16 May 2018.

Casement, S., Flannery, M., Glassman, T., and Lo, A.: Starshade design driven by stray light from edge scatter, Proc. SPIE, $84424 \mathrm{H}$, https://doi.org/10.1117/12.926933, 2012.

Swanner, N.: Leather Tablet Case, Kernel Description, available at: https://www.slashgear.com/ moshi-versacover-review-origami-cool-for-the-ipad-22380289/ (last access: 16 May 2018), 2015.

Tachi, T.: Geometric considerations for the design of rigid origami structures, in: Proceedings of the International Association for Shell and Spatial Structures (IASS) Symposium, 12, 458-460, 2010.
Tachi, T. and Hull, T. C.: Self-Foldability of Rigid Origami, J. Mech. Robot., 9, 021008, https://doi.org/10.1115/DETC201660546, 2017.

Thrall, A. and Quaglia, C.: Accordion shelters: A historical review of origami-like deployable shelters developed by the US military, Eng. Struct., 59, 686-692, 2014.

Thün, G., Velikov, K., Ripley, C., Sauvé, L., and McGee, W.: Soundspheres: resonant chamber, Leonardo, 45, 348-357, 2012.

Waitukaitis, S., Menaut, R., Chen, B. G.-G., and van Hecke, M.: Origami multistability: From single vertices to metasheets, Phys. Rev. Lett., 114, 055503 , https://doi.org/10.1103/PhysRevLett.114.055503, 2015.

Wee, D.: Bao Bao Issey Miyake Launches New “Distortion” Series, available at: http://www.blouinartinfo.com/news/story/1038919/ bao-bao-issey-miyake-launches-new-distortion-series (last access: 16 May 2018), 2014.

$\mathrm{Wu}, \mathrm{W}$. and You, Z.: Modelling rigid origami with quaternions and dual quaternions, P. Roy. Soc. A-Math. Phy., 466, 2155-2174, 2010.

$\mathrm{Wu}, \mathrm{W}$. and You, Z.: A solution for folding rigid tall shopping bags, P. Roy. Soc. A-Math. Phy., 467, 2561-2574,2011.

Yang, L.: These futuristic origami-style utensils should be in every kitchen - check them out, available at: https://www.businessinsider.com/ origami-inspired-kitchen-utensils-kickstarter-2017-4?IR=T (last access: 16 May 2018), 2017.

Yang, Y., Nara, C., Chen, X., and Hagiwara, I.: Investigation of Helmet Based on Origami Structures, in: ASME 2017 International Design Engineering Technical Conferences and Computers and Information in Engineering Conference, American Society of Mechanical Engineers, V05BT08A040, 2017.

Yong, J.: A Furniture Set That Can Be Folded Like Origami Paper, available at: http://designtaxi.com/news/358580/ A-Furniture-Set-That-Can-Be-Folded-Like-Origami-Paper/ (last access: 16 May 2018), 2013.

Zirbel, S. A., Lang, R. J., Thomson, M. W., Sigel, D. A., Walkemeyer, P. E., Trease, B. P., Magleby, S. P., and Howell, L. L.: Accommodating thickness in origami-based deployable arrays, J. Mech. Design, 135, 111005, https://doi.org/10.1115/1.4025372, 2013. 